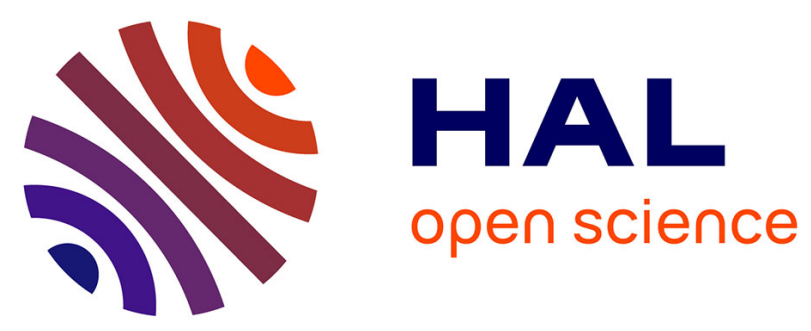

\title{
Interatomic Coulombic Electron Capture from first principles
}

Nicolas Sisourat, Tsveta Miteva, Jimena D Gorfinkiel, Kirill Gokhberg, Lorenz S Cederbaum

\section{- To cite this version:}

Nicolas Sisourat, Tsveta Miteva, Jimena D Gorfinkiel, Kirill Gokhberg, Lorenz S Cederbaum. Interatomic Coulombic Electron Capture from first principles. Physical Review A : Atomic, molecular, and optical physics [1990-2015], 2018, 98 (2), pp.020701. 10.1103/PhysRevA.98.020701 . hal-01905815

\section{HAL Id: hal-01905815 https://hal.sorbonne-universite.fr/hal-01905815}

Submitted on 26 Oct 2018

HAL is a multi-disciplinary open access archive for the deposit and dissemination of scientific research documents, whether they are published or not. The documents may come from teaching and research institutions in France or abroad, or from public or private research centers.
L'archive ouverte pluridisciplinaire HAL, est destinée au dépôt et à la diffusion de documents scientifiques de niveau recherche, publiés ou non, émanant des établissements d'enseignement et de recherche français ou étrangers, des laboratoires publics ou privés. 


\title{
Interatomic Coulombic Electron Capture from first principles
}

\author{
Nicolas Sisourat, ${ }^{1, *}$ Tsveta Miteva, ${ }^{1}$ Jimena D. \\ Gorfinkiel, ${ }^{2}$ Kirill Gokhberg, ${ }^{3}$ and Lorenz S. Cederbaum ${ }^{3}$ \\ ${ }^{1}$ Sorbonne Université, CNRS, Laboratoire de \\ Chimie Physique Matière et Rayonnement, \\ UMR 7614, F-75005 Paris, France \\ ${ }^{2}$ School of Physical Sciences, The Open University, \\ Walton Hall, Milton Keynes, MK'7 6AA, United Kingdom. \\ ${ }^{3}$ Theoretische Chemie, Physikalisch-Chemisches Institut, Universität Heidelberg, \\ Im Neuenheimer Feld 229, Heidelberg D-69120, Germany.
}

(Dated: October 13, 2018)

\begin{abstract}
Interatomic Coulombic Electron Capture (ICEC) is an environment assisted process in which a free electron can efficiently attach to an ion, atom or molecule by transferring the excess energy to a neighboring species. Absolute cross sections are necessary to evaluate the relative importance of this process. In this work, we employ the R-Matrix method to compute ab initio these cross sections for a singly charged neon ion embedded in small helium clusters. Our results show that the ICEC cross sections are several orders of magnitude higher than anticipated and dominate other competing processes. Electron energy loss spectra on an absolute scale are provided for $\mathrm{Ne}^{+} @ \mathrm{He}_{20}$ cluster. Such spectra exhibit an unambiguous signature of the ICEC process. The finding is expected to stimulate experimental observations.
\end{abstract}

\footnotetext{
* nicolas.sisourat@upmc.fr
} 
Free electrons can attach themselves to ions, atoms and molecules through radiative recombination [1] (also known as radiative attachment) or through rovibrational excitation and dissociative electron attachment (see [2-4] and references therein) in the case of molecules. Such electron capture processes play a central role in plasma physics [5], astrophysics [6], environmental science [7] and radiation damage [8-10]. It was recently predicted that electron attachment to a species can be strongly enhanced in the presence of a chemical environment through the so called Interatomic Coulombic Electron Capture (ICEC) mechanism [11, 12]. In the latter, the excess energy of the electron attachment is transferred to a neighbor which is thus ionized. A strong enhancement of the electron capture cross sections was demonstrated using an analytical formula which is only valid in the limit of large distances between the neighbors. Therefore, a complete picture of ICEC at different interatomic distances is still missing. Relevant open questions are at what distances the analytical formula applies and how the cross sections behave at smaller distances. Furthermore, to date ICEC processes have only been investigated theoretically and an experimental evidence remains to be provided. The present investigation of experimentally relevant clusters should help in designing future experiments.

In this Letter, we report on the first ab initio calculations of absolute ICEC cross sections. Experimentally relevant interatomic distances and geometries are investigated. On the example of singly charged neon ion embedded in helium clusters (i.e. $\mathrm{Ne}^{+} @ \mathrm{He}, \mathrm{Ne}^{+} @ \mathrm{He}_{2}$ and $\mathrm{Ne}^{+} @ \mathrm{He}_{20}$ ), we show that for these interatomic distances the ICEC cross sections are nearly two orders of magnitude larger than predicted by the asymptotic formula and four to five orders of magnitude larger than radiative recombination cross sections. Furthermore, our calculations show that the ICEC cross sections increase nearly linearly with the number of neighbors. We also provide cross sections for realistic systems which should help the experimental observation of ICEC.

The ICEC cross sections were computed using the R-Matrix method as implemented in the UKRmol package. A review of the R-Matrix method can be found in [13] and details of the UKRmol package are reported in [14]. Here we only summarize the method and the implementation used in this work.

In the R-Matrix method, the configuration space is partitioned into an inner and an outer region separated by a sphere of radius $a$ centered at the center of mass of the system. The inner region contains the multielectron description of the full system composed of $N+1$ 
electrons. In the outer region only the free electron is treated and the interaction with the $\mathrm{N}$ remaining electrons is described in terms of a multipole expansion. The R-matrix links the two regions. Analysis of the wavefunction in the outer-region provides elastic and inelastic cross sections.

The calculations on $\mathrm{Ne}^{+}-\mathrm{He}$ and $\mathrm{Ne}^{+}-\mathrm{He}_{2}$ in this work were performed using the same parameters as those reported in [15]: we used the cc-pVDZ basis set and the HartreeFock (HF) orbitals optimized for the neutral clusters using the MOLPRO package [16, 17]. The R-matrix radius was fixed at 13 a.u. and we employed 151 continuum-like orbitals with angular momemtum up to $l=4$. The electronic wavefunction in the inner region is constructed including the target states corresponding to the $\mathrm{Ne}^{+}\left(2 p^{-1}\right)$ and the $\mathrm{He}^{+}\left(1 s^{-1}\right)$ states. These states are described as single determinants formed by removing one electron from the corresponding orbital in the $\mathrm{HF}$ ground state determinant. Furthermore, the lowest configurations of $\mathrm{NeHe}\left(\right.$ or $\mathrm{NeHe}_{2}$ ) in each spatial symmetry were included as $L^{2}$ configurations. For the outer region calculations, the R-matrix is propagated from $a=13$ a.u. to 80 a.u. which is sufficient for obtaining converged cross sections. The maximum multipole retained in the expansion of the long range potential was set to 2 .

In the limit of large interatomic distances $(R)$, the ICEC cross sections for $\mathrm{Ne}^{+}-\mathrm{He}$ can be obtained from the photoionization cross sections of atomic neon and helium $\left(\sigma_{\mathrm{PI}}^{(\mathrm{Ne})}\right.$ and $\left.\sigma_{\mathrm{PI}}^{(\mathrm{He})}\right)$ according to $[11,12]$

$$
\sigma_{\mathrm{ICEC}}(e)=\frac{3 \hbar^{4} c^{2}}{8 \pi m_{e}} \frac{g_{\mathrm{Ne}}}{g_{\mathrm{Ne}^{+}}} \frac{\sigma_{\mathrm{PI}}^{(\mathrm{Ne})}(e) \sigma_{\mathrm{PI}}^{(\mathrm{He})}\left(e^{\prime}\right)}{e R^{6} E_{\mathrm{vph}}^{2}}
$$

where $e$ and $e^{\prime}$ are the energies of the incoming and outgoing electrons, respectively, i.e., of the electron impinging on $\mathrm{Ne}^{+}$and of the electron emitted from He. The statistical weights of the quantum states are $g_{\mathrm{Ne}}=1$ and $g_{\mathrm{Ne}^{+}}=6$. The energy transferred between the species is $E_{\mathrm{vph}}=\mathrm{IP}_{\mathrm{Ne}}+e$ with $\mathrm{IP}_{\mathrm{Ne}}=21.56 \mathrm{eV}$ the ionization potential of neon. The atomic photoionization cross sections were taken from [18]. The cross sections from Eq. 1 are compared to those from the R-Matrix calculations below.

Figure 1 shows the absolute ICEC cross sections (in Mb) as functions of the electron energy $(e)$ for $\mathrm{Ne}^{+}-\mathrm{He}$ at the interatomic distance $\mathrm{R}=4$ a.u., which is about the equilibrium distance of $\mathrm{Ne}^{+}-\mathrm{He}$ dimer. The calculated ICEC cross sections for all electron energies are nearly two orders of magnitude larger than those given by the analytical formula. Moreover, it is also seen that the cross sections decrease more slowly at higher electron energies than 


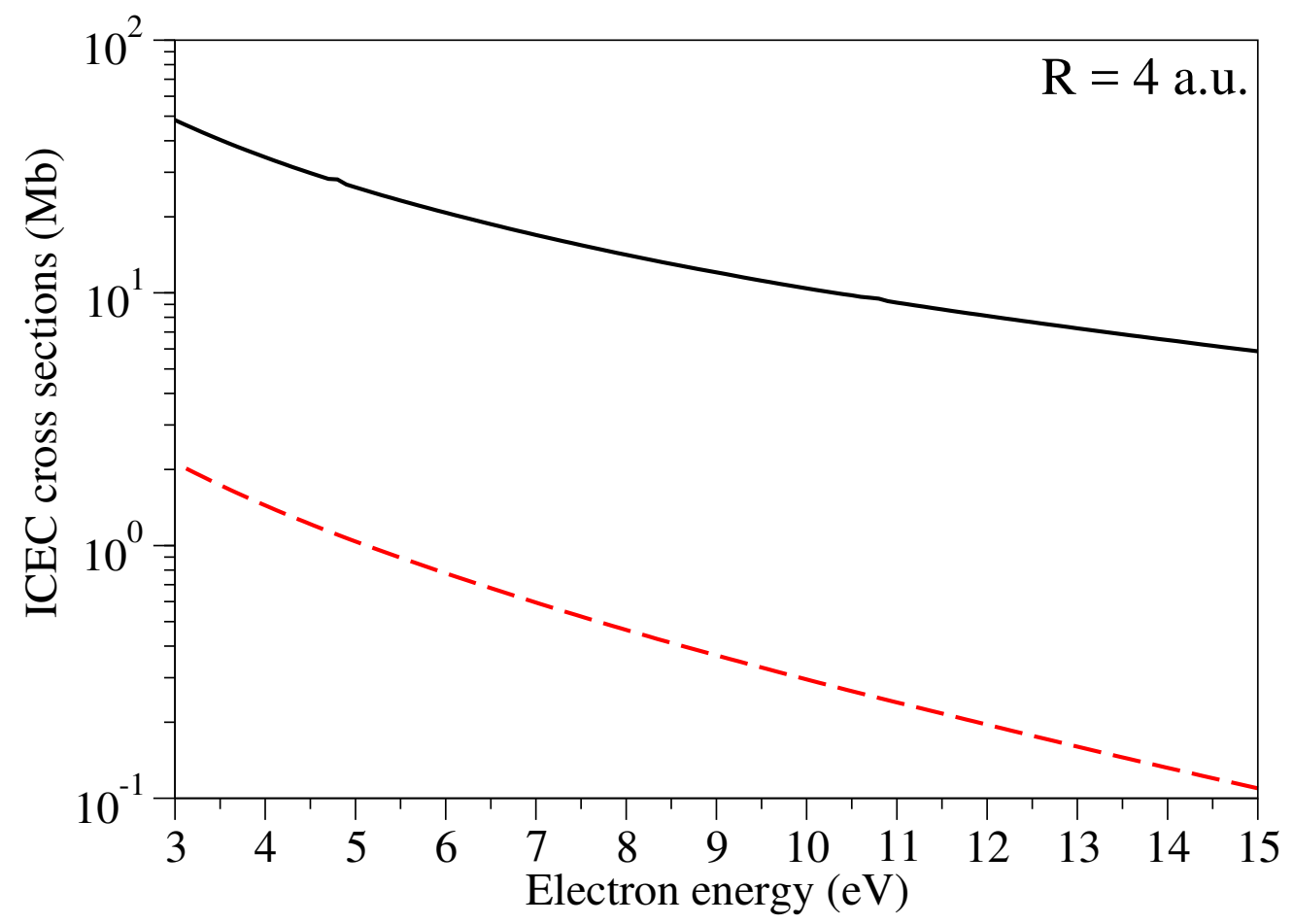

FIG. 1. Absolute ICEC cross sections (in $\mathrm{Mb}$ ) as functions of the electron energy for $\mathrm{Ne}^{+}-\mathrm{He}$ at the interatomic distance $R=4$ a.u. This value is about the equilibrium interatomic distance of $\mathrm{Ne}^{+}-\mathrm{He}$. The full black line represents results from the R-Matrix calculations and the red dashed line the cross sections given by the asymptotic formula (see text).

the asymptotic formula predicts. In the derivation of the latter, the overlaps between the orbitals of $\mathrm{Ne}$ and $\mathrm{He}$ are neglected, as is indeed the case at larger distances. The ab initio results demonstrate that these orbital overlaps substantially enhance the ICEC efficiency at typical cluster distances.

An isolated atom or atomic ion can only capture an electron via photorecombination. For further comparison, we mention that the photorecombination cross section of $\mathrm{Ne}^{+}$in this electron energy range is between $10^{-3}$ and $10^{-4} \mathrm{Mb}[12]$, which demonstrates the great efficiency of the ICEC process.

To determine the range of validity of the asymptotic formula (Eq. 1) we further investigate the ICEC process by varying the interatomic distance for chosen values of the energy of the incoming electron. We chose two representative electron energies, namely 5 and 10 eV. Fig. 


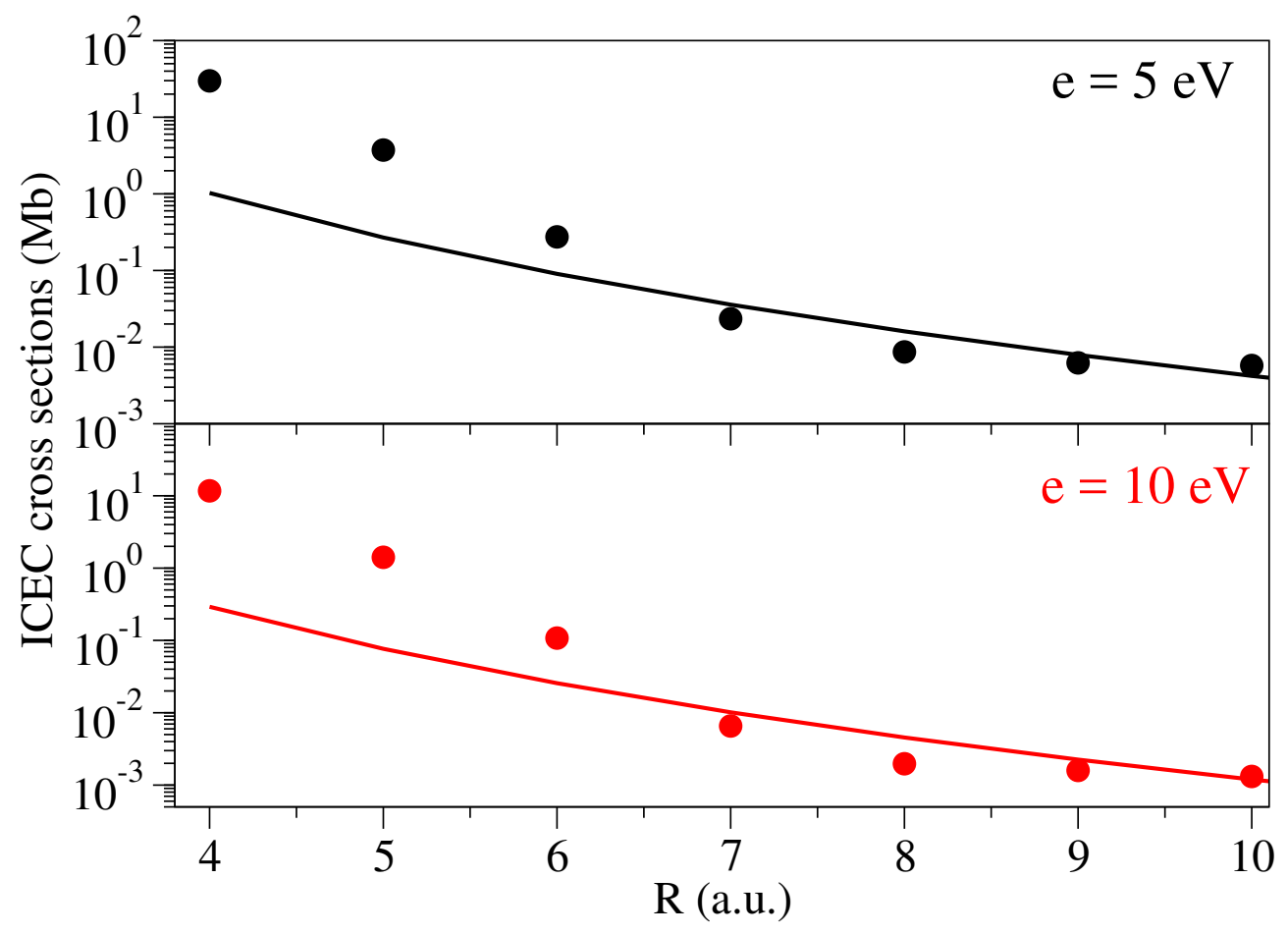

FIG. 2. Absolute ICEC cross sections (in Mb) as functions of the interatomic distances $(R)$ for $\mathrm{Ne}^{+}$-He. Top panel: electron energy e $=5 \mathrm{eV}$. Bottom panel: electron energy e $=10 \mathrm{eV}$. The circles represent results from the R-Matrix calculations and the lines report the cross sections given by the asymptotic formula (see text).

2 shows the absolute ICEC cross sections as functions of $R$. For both electron energies, the analytical formula and the R-Matrix calculations predict similar ICEC cross sections for distances above 7 a.u. It is worth noting that the R-Matrix calculations reproduce well the asymptotic behavior of the ICEC cross sections. However, above $R=10$ a.u. the R-Matrix calculations become unstable owing to the finite size of the basis set. Below $R=7$ a.u. the cross sections from the R-Matrix calculations increase much faster than $R^{-6}$.

It should be mentioned that the asymptotic limit is reached in this system at an exceptionally short interatomic distance of 7 a.u. owing to the rather small atomic radii of neon and helium, which are the most inert atoms available. We have performed similar calculations for other rare gas dimers which show that the ICEC cross sections are much higher than predicted by the asymptotic formula over a larger range of interatomic distances. The 
onset of applicability of the asymptotic formula is in general at rather large distances and the high efficiency of the ICEC process found here is expected to be generic.

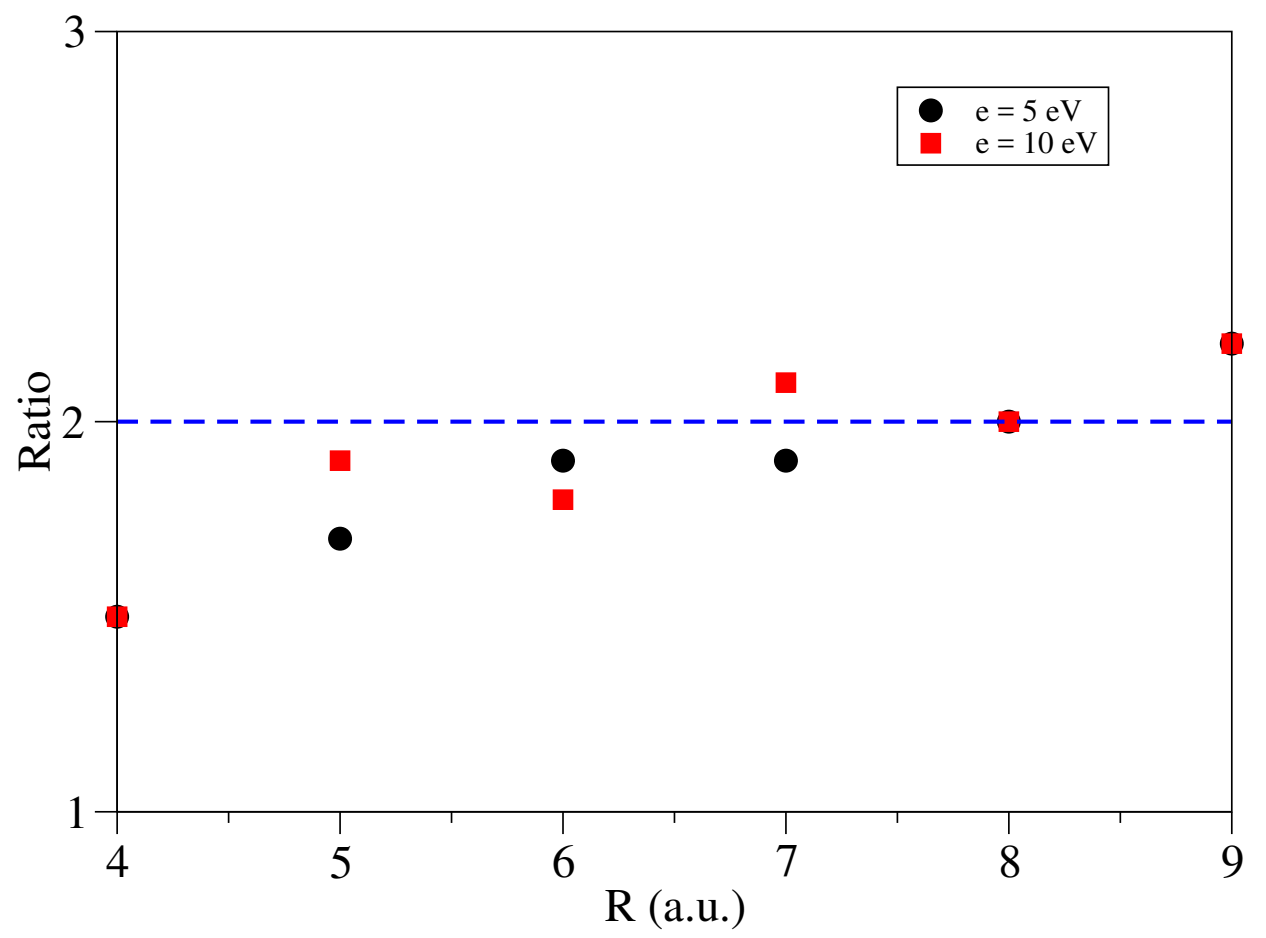

FIG. 3. Ratio between the ICEC cross sections computed for $\mathrm{Ne}^{+}-\mathrm{He}$ and $\mathrm{He}_{-} \mathrm{Ne}^{+}-\mathrm{He}$ as functions of the Ne-He interatomic distances $(R)$. Black circles: electron energy e $=5 \mathrm{eV}$. Red squares: electron energy e $=10 \mathrm{eV}$. Note that the ratios at $R=4,8$, and 9 a.u. are on top of each other. The blue dashed line shows the expected ratio in case of strict additivity. Strict additivity is expected at the distances at which the asymptotic formula (Eq. 1) is valid.

We have so far considered the ICEC process in the presence of only one neighbor. If several helium atoms are in proximity to the neon ion, any one of them can be ionized via ICEC. In the asymptotic limit, the total ICEC cross sections are given by the sum over all $\mathrm{Ne}^{+}-\mathrm{He}$ pairs contributing to ICEC. In order to check the validity of this additivity approximation by ab initio computations, we compare, for the same Ne-He distances the ICEC cross sections for $\mathrm{Ne}^{+}-\mathrm{He}$ with that of $\mathrm{Ne}^{+}-\mathrm{He}_{2}$ where the ion is centered between the two helium atoms. The ratio between the corresponding cross sections is shown in Fig. 3: for both electron energies, the ratios are approximately two. 
As a first approximation, the total ICEC cross sections for $\mathrm{Ne}^{+}$embedded in a helium cluster can therefore be estimated from the cross sections computed for the dimer. Using these cross sections as input data, we were able to compute the total ICEC cross sections for $\mathrm{Ne}^{+} @ \mathrm{He}_{20}$ which provides a realistic system for which ICEC could be measured (see below for details). Experimentally, electron energy loss spectroscopy [19-21] would be particularly well suited for investigating ICEC in $\mathrm{Ne}^{+}$embedded in helium clusters. Since for low energy electrons ICEC is the only allowed electronic process, one can prove the ICEC by measuring the typical energies of the electrons emitted from the He environment and/or the possible appearance of $\mathrm{He}^{+}$ions in spite of the impact with electrons of just a few electron volt energy.

To compute the total ICEC cross section of the cluster and the distribution of the energies of the emitted electrons, one needs in addition to the individual cross sections of the $\mathrm{Ne}^{+}$ He pairs as function of $R$, the nuclear wavefunction of the whole cluster. The wavefunction for $\mathrm{Ne}^{+} @ \mathrm{He}_{20}$ was obtained from a variational quantum Monte Carlo calculation [22]. The wavefunction ansatz was chosen as in [23]:

$$
\Phi=\chi^{\mathrm{Ne}}(r) \prod_{i} \chi^{\mathrm{He}}\left(r_{i}\right) \phi^{\mathrm{Ne}-\mathrm{He}}\left(r_{i \mathrm{Ne}}\right) \prod_{i<j} \phi^{\mathrm{He}-\mathrm{He}}\left(r_{i j}\right)
$$

where $r$ and $r_{i}$ indicate the positions, from the center of mass of the cluster, of neon and helium atom $i$, respectively. The interatomic distances between neon and one helium atom and between two helium atoms are given by $r_{i \mathrm{Ne}}$ and $r_{i j}$, respectively. The one-body terms are

$$
\chi^{I}(r)=e^{-\frac{1}{2 b^{2}} r^{2}}
$$

and the two-body terms are given by

$$
\phi^{I-J}\left(r_{i j}\right)=e^{-\frac{1}{2}\left(\frac{a}{r_{i j}}\right)^{5}} .
$$

The parameters $a$ and $b$ in the terms involving only helium atoms were taken from [23]. For the terms linked to neon, these parameters were optimized in order to minimize the electronic energy. We found $a=2.0 \AA$ and $b=5.0 \AA$. The potential energy was approximated as a sum of pair potentials. The $\mathrm{He}-\mathrm{He}$ and $\mathrm{Ne}^{+}-\mathrm{He}$ pair potentials were taken from [24] and [25], respectively.

Using the data from the R-Matrix calculations and the nuclear wavefunction described above the electron energy loss spectra for two electron energies ( $e=5$ and $10 \mathrm{eV})$ were 
computed: 2000 geometries which sample the square of the wavefunction were obtained with a Metropolis-Hastings sampling technique. For each geometry, we computed the ICEC crosssection as the sum over all $\mathrm{Ne}^{+}-\mathrm{He}$ pairs. The computed energy loss spectra of $\mathrm{Ne}^{+} @ \mathrm{He}_{20}$ are depicted in Fig. 4. Although these spectra can be measured by electron energy loss spectroscopy, strictly speaking they are not energy loss spectra, but rather ICEC spectra: the incoming electrons are captured by $\mathrm{Ne}^{+}$and the electrons emitted from the He environment are measured. The spectra exhibit two peaks (energy loss of 0 and $3.1 \mathrm{eV}$ ). The peaks at $0 \mathrm{eV}$ correspond to the elastic peak computed with the R-matrix method. The calculations show that the elastic cross sections depend only weakly on the number of neighbors and the distribution of the distances between them and the ion. For simplicity, we therefore, used the elastic cross sections computed for $\mathrm{Ne}^{+}-\mathrm{He}$ dimer at $R=4$ a.u.

The ICEC cross sections, computed with the R-Matrix method, are equal to about 7 and $3 \mathrm{Mb}$ for $e=5$ and $10 \mathrm{eV}$, respectively. In both cases, they are higher by nearly two orders of magnitude than predicted by the asymptotic formula, and four to five orders of magnitude higher than the radiative recombination cross sections. It should also be noted that the ICEC cross sections are only two orders of magnitude smaller than the elastic ones, illustrating quantitatively the ICEC efficiency.

In conclusion, we employed the R-matrix method to compute $a b$ initio the ICEC cross sections in $\mathrm{Ne}^{+} @ \mathrm{He}_{n}$ clusters with $n=1,2$ and used the results to determine the cross sections for $n=20$ using the corresponding nuclear wavefunction obtained from a variational Monte Carlo calculation. Our results demonstrate that the ICEC cross sections are orders of magnitude higher than anticipated. We provided absolute ICEC cross sections for a realistic system and compared them to the elastic cross sections. Such quantitative data should help the experimental observation of ICEC.

The Ne and He atoms studied here are the most inert atoms known. In spite of this fact, we found that the ab initio ICEC cross sections are much larger than predicted by the asymptotic formula. This is due to orbital overlap. This in turn implies that we may expect even larger enhancements for less inert participants. First numerical results in this direction support this expectation. The asymptotic formula can be considered as a lower bound for the ICEC cross section. In some of the examples studied in $[11,12]$ using the asymptotic formula, particularly large cross sections have been predicted. This supports the expectation that the true cross sections can be very large. 


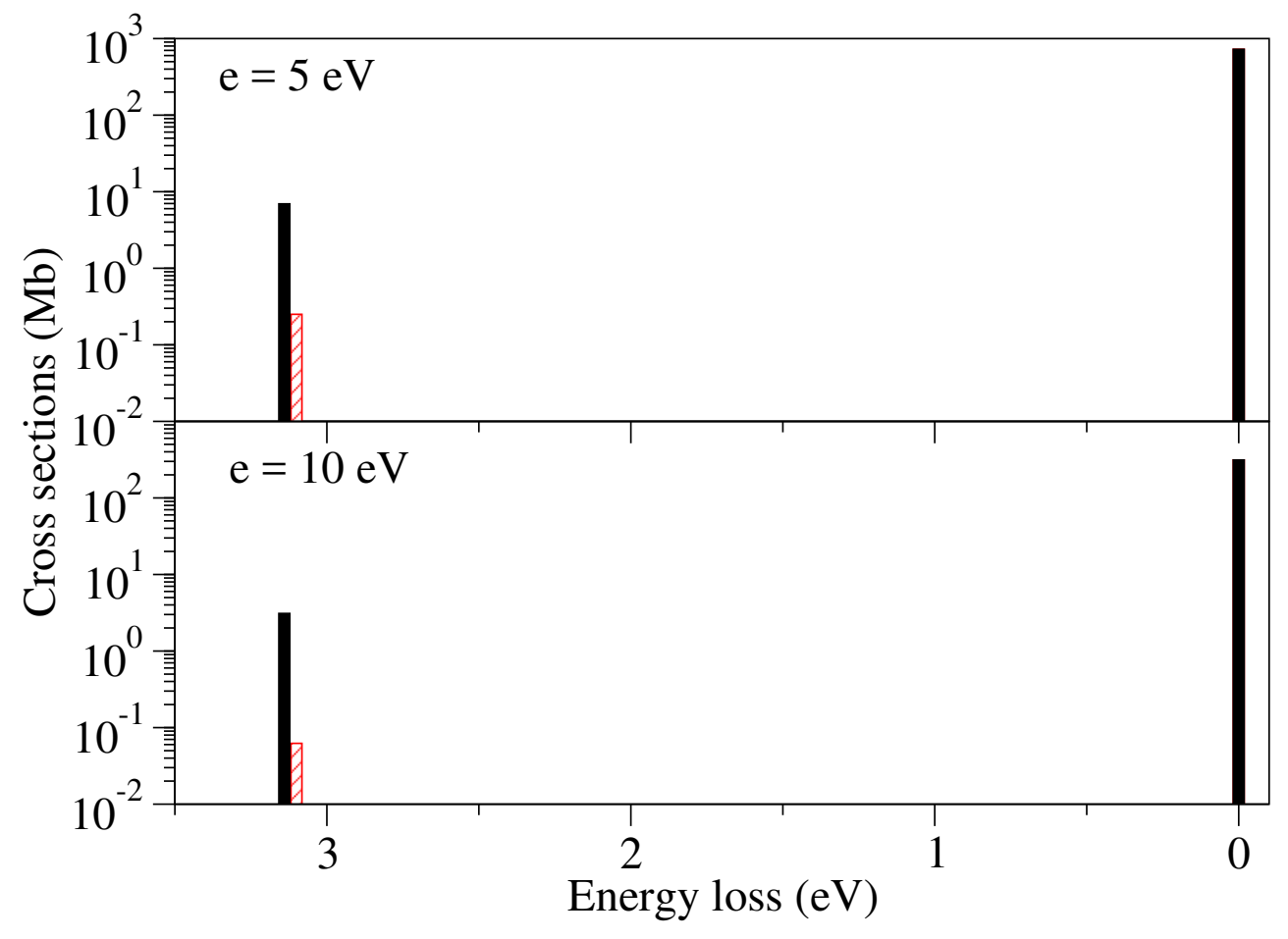

FIG. 4. Energy loss (or ICEC) spectra (in Mb) for $\mathrm{Ne}^{+} @ \mathrm{He}_{20}$. Upper panel: the incoming electron energy is $5 \mathrm{eV}$. Lower panel: the incoming electron energy is $10 \mathrm{eV}$. The spectra are determined using the computed nuclear wavefunction of the whole cluster and the computed individual ICEC cross sections of all $\mathrm{Ne}^{+}$-He pairs. The small energy broadening due to the distribution of interatomic distances was neglected. Full black bars report the ab initio R-Matrix calculations. Red dashed bars show results obtained employing the asymptotic formula. Note that the energy of the incoming electron is too low to cause excitations in $\mathrm{Ne}^{+}$or in $\mathrm{He}$.

Since electron capture by ions, atoms and molecules represents the first fundamental step in many field of physics, ICEC may play an important role and should be considered in future theoretical and experimental studies.

This project has received funding from the Research Executive Agency (REA) under the European Union's Horizon 2020 research and innovation programme Grant agreement No. 705515 and from Agence Nationale de la Recherche through the program ANR-16CE29-0016-01. Financial support by the European Research Council (ERC) (Advanced 
Investigator Grant No. 692657) is gratefully acknowledged.

[1] F. L. Mohler, Rev. Mod. Phys. 1, 216 (1929).

[2] E. Illenberger, "Electron attachment to molecules," in Linking the Gaseous and Condensed Phases of Matter: The Behavior of Slow Electrons, edited by L. G. Christophorou, E. Illenberger, and W. F. Schmidt (Springer US, Boston, MA, 1994) pp. 355-376.

[3] I. I. Fabrikant, J. of Phys.: Conf. Ser. 204, 012004 (2010).

[4] I. I. Fabrikant, S. Eden, N. J. Mason, and J. Fedor, in Advances In Atomic, Molecular, and Optical Physics, Advances In Atomic, Molecular, and Optical Physics, Vol. 66, edited by C. C. L. Ennio Arimondo and S. F. Yelin (Academic Press, 2017) pp. 545 - 657.

[5] A. Chutjian, A. Garscadden, and J. Wadehra, Phys. Rep. 264, 393 (1996).

[6] T. J. Millar, C. Walsh, and T. A. Field, Chem. Rev. 117, 1765 (2017).

[7] Q.-B. Lu and L. Sanche, Phys. Rev. Lett. 87, 078501 (2001).

[8] B. Boudaïffa, P. Cloutier, D. Hunting, M. A. Huels, and L. Sanche, Science 287, 1658 (2000).

[9] E. Alizadeh, T. M. Orlando, and L. Sanche, Ann. Rev. Phys. Chem. 66, 379 (2015).

[10] L. Sanche, Rwd. Phys. Chem. 128, 36 (2016).

[11] K. Gokhberg and L. S. Cederbaum, J. Phys. B: Atomic, Molecular and Optical Physics 42, 231001 (2009).

[12] K. Gokhberg and L. S. Cederbaum, Phys. Rev. A 82, 052707 (2010).

[13] J. Tennyson, Phys. Rep. 491, 29 (2010).

[14] J. Carr, P. Galiatsatos, J. Gorfinkiel, A. Harvey, M. Lysaght, D. Madden, Z. Mašin, M. Plummer, J. Tennyson, and H. Varambhia, Eur. Phys. J. D 66, 58 (2012).

[15] N. Sisourat, S. Engin, J. D. Gorfinkiel, S. Kazandjian, P. Kolorenč, and T. Miteva, J. Chem. Phys. 146, 244109 (2017).

[16] H.-J. Werner, P. J. Knowles, G. Knizia, F. R. Manby, and M. Schütz, WIREs Comput Mol Sci 2, 242 (2012).

[17] H.-J. Werner, P. J. Knowles, G. Knizia, F. R. Manby, M. Schütz, P. Celani, W. Györffy, D. Kats, T. Korona, R. Lindh, A. Mitrushenkov, G. Rauhut, K. R. Shamasundar, T. B. Adler, R. D. Amos, A. Bernhardsson, A. Berning, D. L. Cooper, M. J. O. Deegan, A. J. Dobbyn, F. Eckert, E. Goll, C. Hampel, A. Hesselmann, G. Hetzer, T. Hrenar, G. Jansen, 
C. Köppl, Y. Liu, A. W. Lloyd, R. A. Mata, A. J. May, S. J. McNicholas, W. Meyer, M. E. Mura, A. Nicklass, D. P. O’Neill, P. Palmieri, D. Peng, K. Pflüger, R. Pitzer, M. Reiher, T. Shiozaki, H. Stoll, A. J. Stone, R. Tarroni, T. Thorsteinsson, and M. Wang, "Molpro, version 2015.1, a package of ab initio programs," (2015).

[18] G. Marr and J. West, Atomic Data and Nuclear Data Tables 18, 497 (1976).

[19] A. Burose, C. Becker, and A. Ding, Z. Phys. D 20, 35 (1991).

[20] M. Allan, J. Phys. B 26, L73 (1993).

[21] H. Kubotera, S. Sakai, T. Sekitsuka, T. Tachibana, and T. Hirayama, Appl. Surf. Sc. 256, 1046 (2009), vASSCAA-4.

[22] D. Blume, M. Lewerenz, F. Huisken, and M. Kaloudis, J. Chem. Phys. 105, 8666 (1996).

[23] S. A. Chin and E. Krotscheck, Phys. Rev. B 45, 852 (1992).

[24] K. T. Tang and J. P. Toennies, J. Chem. Phys. 118, 4976 (2003).

[25] D. Bonhommeau, M. Lewerenz, and N. Halberstadt, J. Chem. Phys. 128, 054302 (2008). 